\title{
A simple micromechanical approach to predict mechanical behaviour of polypropylene/organoclay nanocomposites based on representative volume element (RVE)
}

\author{
Yu Dong ${ }^{\mathrm{a},}$, Debes Bhattacharyya ${ }^{\mathrm{b}}$ \\ a Department of Mechanical Engineering, Curtin University of Technology, Perth, \\ WA 6845, Australia \\ ${ }^{\mathrm{b}}$ Centre for Advanced Composite Materials, The University of Auckland, Private Bag \\ 92019, Auckland, New Zealand
}

\begin{abstract}
Two-dimensional $3 \times 3$ array representative volume element (RVE) models, using finite element analysis (FEA), have been employed to predict the elastic moduli $\left(E_{c}\right)$ of polypropylene (PP)/organoclay nanocomposites in terms of clay contents, clay aspect ratios and their dispersion patterns. Three-phase RVE models incorporating the interphase between clay platelets and the PP matrix demonstrate significant modulus enhancement in the exfoliated nanocomposites. The interphase properties have less impact on the moduli of exfoliated nanocomposites while those of intercalated nanocomposites are quite independent of clay dispersion patterns. The numerical results are finally compared with composites theories and experimental data for validity.
\end{abstract}

Keywords: polymer matrix composites (PMCs); representative volume element (RVE); elastic properties; interphase; finite element analysis (FEA); nanostructures.

* Corresponding author. Tel.: +61 892669055 ; fax: +61 892662681.

E-mail address: Y.Dong@curtin.edu.au (Y. Dong). 


\section{Introduction}

Polymer/clay nanocomposites including well dispersed clay platelets with at least one dimension smaller than $100 \mathrm{~nm}$ are considered to be one of the most innovative composite materials. Such nanocomposites benefit from the enhancement of mechanical properties such as higher modulus and strength [1, 2], good thermal stability [3], fire retardancy [3, 4] as well as excellent barrier properties [5] owing to the tortuous pathway in constituent structures. In an ideal situation, two typically categorised morphologies of nanocomposites are formed, which are known as intercalated and exfoliated structures, Fig. 1. When the polymer chains are inserted into stack-layered formation of clay platelets and broaden their interlayer spacing, a well-ordered multilayer intercalated structure occurs consisting of alternating polymeric molecules and clay platelets. In the exfoliated structure, individual clay platelets are completely peeled apart and uniformly dispersed into the continuous polymer matrix

Although the development of polyamide /clay nanocomposites has been pioneered by Toyota R\&D groups [1] and successfully grown in recent years, market-driving industries tend to utilise the polyolefins such as polypropylene (PP) and polyethylene (PE) for their automotive and packaging applications. Consequently, the development of PP/organoclay nanocomposites [6-8] has drawn close attention from the researchers and engineers. However, the incompatibility and low interactions between hydrophobic PP and hydrophilic clay become very formidable. Recent studies $[6,8]$ have shown that the use of functional maleic anhydride grafted PP (MAPP) as the compatibiliser can improve the clay dispersion with more effective intercalated structures. 
Due to the complex nanoscale composite system, the stiffness enhancement of nanocomposites depends mainly on the clay aspect ratio and clay content, morphological structures in different dispersion states and sometimes the presence and amount of compatibilisers, especially for polyolefin/clay nanocomposites. The modulus prediction has been carried out based on analytical techniques using the conventional composite models, finite element analysis (FEA) and molecular dynamics (MD) simulation. Well-known Halpin-Tsai [9] and Mori-Tanaka models [10] have been initially applied to simulate the exfoliated nylon6/clay nanocomposites with quite satisfactory results $[11,12]$. Moreover, Hui-Shia model $[13,14]$ has been employed to consider the elastic moduli of unidirectionally aligned composites with perfect or imperfect interfacial bondings for elastomer/clay nanocomposites.

A two-dimensional numerical simulation was also successfully performed using FEA in well aligned RVE patterns $[12,15]$, which provided a better agreement with the experimental data compared to the conventional Mori-Tanaka and Halpin-Tsai models. MD simulation, on the other hand, can predict more accurately the mechanical properties $[16,17]$ down to the nanoscale level but might get constrained by the integrated large lengths and time scales in the structural analysis of nanocomposites when the scale-up scheme is implemented.

Previous work has basically dealt with the numerical simulation of exfoliated nanocomposite structures which appear difficult to achieve in PP/organoclay nanocomposites due to the incompatibility problem between the PP matrix and clay particles. Most likely, PP/organoclay nanocomposites are highly intercalated with a limited amount of exfoliated structures. The selection of compatibiliser MAPP [18] can also affect the interphase properties of such nanocomposites since MAPP molecular chains are highly mobilised around the clay platelets. The aim of this work 
is to specifically evaluate the reinforcement efficiency of PP/organoclay nanocomposites due to the clay content and clay aspect ratio, interphase properties and dispersion pattern using RVE numerical modelling approach for understanding the validity of conventional composite theories.

\section{Numerical models to predict elastic moduli}

\subsection{Modelling parameters}

The commercial finite element package, ANSYS ${ }^{\circledR}$ was used to construct the RVE models on a continuum scale. Due to the great dimensional variations between the continuous polymer matrix and clay platelets with different aspect ratios in the range of 10-1000, 2-D RVE models have been found to be sufficient since their cross section can give a good representation of clay platelets in a 3-D domain [12, 15]. For simplicity, it can be assumed that clay platelets are unidirectionally well-aligned and uniformly dispersed into the polymer matrix. The selected modelling parameters comprise clay aspect ratio, clay content, clay platelet distribution pattern and the interphase properties between clay platelets and the polymer matrix. Furthermore, the aspect ratios have been fixed to be 10 and 100 with the thickness of clay particles $t=10 \mathrm{~nm}$ and $1 \mathrm{~nm}$, respectively, which are defined as two ideal benchmarks of complete intercalation and exfoliation for a nanocomposite system [19].

\subsection{RVE patterns}

It is well understood from focused morphologies of nanocomposites that most clay platelets are randomly distributed with irregular sizes and shapes. In a simplest case of periodic particle structures, three types of RVE patterns have been evaluated, which are denoted as "stack", "staggering" and 'randomly distributed" patterns, as illustrated in Figs. 2(a)-(c). In order to quantify the relative spatial position of clay platelets, a 
staggering factor $f_{s}$ [20] has been introduced to represent the platelet overlap at different staggering level written as

and

$$
\begin{array}{r}
f_{s}=\frac{2 \Delta L}{b} \\
b=\frac{\rho_{f}}{\rho_{m}}\left[\frac{L t}{a}\left(\frac{1}{W_{f}}-1\right)\right]
\end{array}
$$

where $\Delta L$ is the offset between clay platelets in the stack, $a$ and $b$ are the length and the width of a single RVE domain. In this entire RVE modelling work, $a$ has been fixed to be $200 \mathrm{~nm}$ on the basis of a fundamental hypothesis that the minimum RVE size is approximately twice the reinforcement diameter [21, 22], which is equivalent to platelet lateral length $(L=100 \mathrm{~nm})$ in this case. $L$ and $t$ are the length and thickness of single clay platelet. $\rho_{f}$ and $\rho_{m}$ are referred to as the densities of clay particles and the PP matrix, respectively while $W_{f}$ is the clay weight fraction. $c$ is the lateral distance between two clay platelets and expressed as $c=b$ for the stack rows in Fig. 2(a).

\subsection{Constituent properties and boundary conditions}

In the conventional two-phase composite system, the constituents of nanocomposites mainly include clay particles and the polymer matrix. However, previous research $[15,20]$ has shown that there might be a third constituent in between with different material properties, known as "interphase", which plays an essential role in the property enhancement of composites especially when the size of reinforced particles is in the order of nanometer. Consequently, it is worthwhile to understand the interphase effect on the overall effective mechanical properties of RVE models. The interphase properties are thus simplified as the interphase modulus and thickness in this study. The analysis of parametric variation has been undertaken with the interphase modulus $E_{i}$ being $0.5 E_{m}, E_{m}$ and $2 E_{m}$ and the interphase thickness $t_{i}$ equal to $0.5 t, t$ and $2 t$, respectively. 
To further simplify the RVE modelling, a few assumptions could be made including: (i) clay, the polymer matrix and the interphase are all isotropic materials under a small elastic deformation (less than 1\% strain); (ii) the intercalated/exfoliated clay platelets are uniformly dispersed with a well-aligned formation; (iii) perfect interfacial bonding takes place at the interfaces of all the constituents; (iv) the actual discrete stack structure of intercalated clay platelets, known as "effective particles", is then approximated as the ideally isotropic homogenised particles with its elastic modulus of $E_{p}$ and Poisson's ratio of $v_{p}$ [12], Fig. 3. The elastic modulus of intercalated clay platelets can be then estimated by the rule of mixtures (ROM) [23, 24], which is written as

$$
E_{p}=\phi_{M M T} E_{M M T}+\phi_{\text {gallery }} E_{\text {gallery }} \approx \phi_{M M T} E_{M M T} \approx\left(\frac{d_{M M T}}{d_{001}}\right) E_{M M T}
$$

with

$$
E_{\text {gallery }}<<E_{M M T}
$$

where $\phi_{M M T}$ and $\phi_{\text {gallery }}$ are the volume fractions of silicate layers in the stack and gallery space (i.e. interlayer area), respectively. $E_{\text {gallery }}$ is the modulus of the interlayer (intragallery) material, which is expected to be far less than the elastic modulus of silicates $\left(E_{M M T}\right)$ [24]. In addition, $E_{\text {gallery }}$ shows insignificant influence on the overall elastic moduli of nanocomposites especially with a higher modulus ratio between the interlayer material and bulk matrix [19]. $\phi_{M M T}$ can be estimated from the ratio of $d_{M M T}$ $\left(d_{M M T} \approx 1 \mathrm{~nm}\right)$ and the interlayer spacing $d_{001}$ of intercalated clay platelets obtained from X-Ray Diffraction (XRD) scans. Using Eq. (3) and interlayer spacing values of PP-Hom H380F based nanocomposites (weight ratio of organoclay and MAPP, $\mathrm{WR}=1: 2$ ) listed in Table 1 [25], the calculated elastic moduli of intercalated clay platelets at various clay contents are determined accordingly, Fig. 4. $E_{M M T}$ has been assumed to be equal to that of muscovite $\left(E_{M M T}=178 \mathrm{GPa}\right)$ [11], which represents the 
elastic modulus of single exfoliated clay platelet. Apparently, the discrepancy of elastic moduli of intercalated clay platelets is very trivial regardless of the clay content. Hence, the average elastic modulus $E_{p}=48.3 \mathrm{GPa}$ has been assigned in the entire intercalated RVE models.

The Poisson's ratio of intercalated clay platelets are also estimated by a simple mixing rule [24] as follows,

$$
v_{p}=\phi_{M M T} v_{M M T}+\phi_{\text {gallery }} v_{\text {gallery }}
$$

where Poisson's ratios $v_{M M T}=0.20$ [11] and $v_{\text {gallery }}=0.35-0.50$ [24]. In this study, $v_{p}=$ 0.26 has been used due to the insensitivity of Poisson's ratio of clay particles within the range of 0.26-0.32 [24]. The constituent properties of PP/clay nanocomposites are presented in Table 2 for both exfoliated and intercalated structures.

The boundary conditions in virtual tensile tests have been employed subjected to zero $\mathrm{X}$ and $\mathrm{Y}$ displacements on the left boundary (i.e. fully constrained) as well as the applied equivalent strains of $0.05 \%$ and $0.25 \%$ on the right boundary of a RVE model, Fig. 2(a).

\subsection{Calculation of elastic modulus}

The calculation of resulting average stress is derived from the sum of the nodal forces on the right boundary and the elastic modulus $\left(E_{c}\right)$ of a RVE model material follows the Hooke's law given by

$$
E_{c}=\frac{\sigma}{\varepsilon_{0}}=\frac{\sum_{i=1}^{n} F_{i}}{\varepsilon_{0} A}=\frac{\sum_{i=1}^{n} F_{i}}{\varepsilon_{0}(1 \cdot 3 b)}=\frac{\sum_{i=1}^{n} F_{i}}{3 b \varepsilon_{0}}
$$

where $\varepsilon_{0}$ is the applied strain and $\sigma$ is the resulting average stress. $F_{i}$ is the nodal force at node $i$ and $A$ is the cross sectional area of the established $3 \times 3 \mathrm{RVE}$ simulation domain with the assumptions of plane stress and unit thickness. 


\subsection{Finite element mesh and model setup}

The geometries of clay platelets and interphases were generated individually which were then copied and subtracted from the RVE models in the ANSYS ${ }^{\circledR}$ interface in order to separate three different phases between clay platelets, the PP matrix and their interphase. However, the three individual phases did not really adhere to one another and the glue command in ANSYS ${ }^{\circledR}$ Booleans menu was utilised to achieve the hypothesis of the perfect interfacial bonding condition. This command allows the stress transfer analysis between the PP matrix, interphase and clay platelets. The finite element mesh was generated using 2-D 4-node elastic shell elements (SHELL 63). The free mesh tool in ANSYS ${ }^{\circledR}$ was implemented to create the coarse mesh of PP matrix with $10 \times 10 \mathrm{~nm}$ shell elements while the clay platelets and interphases underwent at least one level of mesh refinement. Typical RVE finite element mesh models in the stack pattern $\left(t_{i}=2 t\right)$ are displayed in Figs. 5(a) and (b) to represent the exfoliated structure (1736 elements and 1863 nodes) and the intercalated structure (7756 elements and 7874 nodes), respectively.

\section{Composite theoretical models}

\subsection{Halpin-Tsai model}

Halpin-Tsai model [9] offers a sophisticated composite theoretical analysis in the fibre composites industry to predict the elastic moduli of a unidirectional composite as the function of filler aspect ratio and volume fraction. It can deal with a variety of reinforcement geometries of discontinuous fillers such as fibre-like or flake-like fillers. The Young's modulus of a composite material in Halpin-Tsai model is written as

$$
\frac{E_{c}}{E_{m}}=\frac{1+\zeta \eta \phi_{f}}{1-\eta \phi_{f}}
$$




$$
\eta=\frac{\frac{E_{f}}{E_{m}}-1}{\frac{E_{f}}{E_{m}}+\zeta}
$$

where $E_{c}, E_{f}$ and $E_{m}$ are Young's moduli of composites, fillers and the polymer matrix, respectively; $\phi_{f}$ is the filler volume fraction and $\zeta$ is a shape parameter depending on the filler geometry and loading direction. In particular, $\zeta=2(l / d)$ for fibres or $2(l / t)$ for disk-like platelets. $l, d$ and $t$ are the length, diameter and thickness of dispersed fillers, respectively.

Since 2-D disk-like clay platelets inevitably diminish the unidirectional reinforcement in comparison with 1-D fibre-like fillers, a modulus reduction factor (MRF) for platelet fillers is thus introduced in the modified Halpin-Tsai model as follows

$$
\frac{E_{c}}{E_{m}}=\frac{1+\zeta(M R F) \eta \phi_{f}}{1-\eta \phi_{f}}
$$

\subsection{Hui-Shia model}

Hui-Shia model [13] is employed to predict the elastic moduli of composites with unidirectional aligned platelets for the simple assumption of perfect interfacial bonding between the polymer matrix and platelet fillers, which is given by

$$
\begin{gathered}
\frac{E_{c}}{E_{m}}=\frac{1}{1-\frac{\phi_{f}}{4}\left[\frac{1}{\xi}+\frac{3}{\xi+\Lambda}\right]} \\
\xi=\phi_{f}+\frac{E_{m}}{E_{f}-E_{m}}+3\left(1-\phi_{f}\right)\left[\frac{(1-g) \alpha^{2}-\frac{g}{2}}{\alpha^{2}-1}\right] \\
g=\frac{\pi}{2} \alpha \\
\Lambda=\left(1-\phi_{f}\right)\left[\frac{3\left(\alpha^{2}+0.25\right) g-2 \alpha^{2}}{\alpha^{2}-1}\right]
\end{gathered}
$$


where $\alpha$ is the inverse aspect ratio of dispersed fillers and $\alpha=t / l$ for disk-like platelets $(\alpha \leq 0.1)$

\subsection{Modified rule of mixture (MROM)}

Conventionally, the composite modulus $E_{c}$ can also be estimated by using a modified rule of mixture (MROM) [13] with flake-like fillers shown as

$$
E_{c}=\phi_{f} E_{f}(M R F)+\left(1-\phi_{f}\right) E_{m}
$$

and

$$
\begin{aligned}
& M R F=1-\frac{\ln (u+1)}{u} \\
& u=\frac{1}{\alpha} \sqrt{\frac{\varphi_{f} G}{E_{f}\left(1-\varphi_{f}\right)}}
\end{aligned}
$$

where $G$ is the shear modulus of the polymer matrix.

\section{Results and discussion}

\subsection{Effect of interphase properties on elastic modulus}

The effect of interphase properties was investigated on the basis of a simple stack pattern at a fixed clay content of $5 \mathrm{wt} \%$. It is clearly seen from Figs. 6(a) and (b) that at a given interphase thickness, increasing the interphase modulus leads to the enhancement of relative modulus $E_{c} / E_{m}$. When $E_{i}=E_{m}$, as expected, $E_{c} / E_{m}$ remains at the same level irrespective of the interphase thickness for both exfoliated and intercalated nanocomposites. This can be interpreted by the fact that the interphase becomes only a part of the PP matrix under the perfect bonding assumption in this case. On the other hand, when $E_{i}=2 E_{m}, E_{c} / E_{m}$ is improved with the increase of interphase thickness, particularly in the intercalated RVE models. Conversely, as the interphase modulus becomes half of the matrix modulus, $E_{c} / E_{m}$ shows a declining trend with increasing interphase thickness. It might be worth noting that $E_{i}=2 E_{m}$ and $t_{i}=2 t$ contribute to the maximum modulus enhancement while $E_{i}=0.5 E_{m}$ and $t_{i}=2 t$ offer 
the worst scenario. More interestingly, interphase properties appear to have less significant influence on the exfoliated nanocomposites regardless of $E_{i}=E_{m}$ since $E_{c} / E_{m}$ possesses a similar decreasing or increasing rate of less than $2.5 \%$ by increasing $t_{i}$, as compared to about $10 \%$ for the corresponding intercalated nanocomposites.

In particular, the axial stress and strain distributions of cross section A-A depicted from Fig. 2(a) in exfoliated and intercalated RVE models $\left(t_{i}=2 t\right)$ are illustrated in Figs. 7 and 8, respectively. Both axisymmetric stress and strain distributions are presented along the mid-plane XZ. For the exfoliated RVE models, Fig. 7(a) indicates that clay inclusions and clay/interphase areas evidently experience higher axial stresses, the maximum being over 48 times greater than those around the PP matrix through the transfer of interfacial shear stresses. However, very small variations of the axial stress distribution take place especially when the interphase modulus is equal to or higher than the matrix modulus. Axial strain increases enormously in the PP matrix, but contrarily becomes much smaller around the neighbouring areas of clay particles and interphases when decreasing the interphase modulus, Fig. 7(b). In the intercalated RVE models, as seen from Fig. 8(a), the change of maximum stress between clay particles and the PP matrix appears to gradually drop from 7.4 to 3.8 times with the reduction of interphase modulus from $2 E_{m}$ to $0.5 E_{m}$. In comparison, the load transfer in exfoliated nanocomposites is far more efficient, which inevitably leads to greater modulus and strength enhancements. The axial strains for such intercalated nanocomposites show very similar distributions, becoming independent of the interphase modulus, Fig. 8(b). Nevertheless, the maximum axial strain of the PP matrix in an intercalated RVE model is about 4 times higher than that in an exfoliated counterpart. Since intercalated RVE models result in greater deformations in the large areas of the PP matrix as well as relatively small axial stresses in clay inclusions, the 
modulus enhancement cannot be superior to that in an exfoliated structure as expected.

\subsection{Effect of staggering factor on elastic modulus}

Illustrated from Figure 9, the staggering factor $f_{s}$ plays a more important role in the reinforcement efficiency of exfoliated nanocomposites compared to those of intercalated ones with the fixed $W_{f}=5 \mathrm{wt} \%$ and $t_{i}=2 t$. As expected, increasing the interphase modulus tends to promote $E_{c} / E_{m}$ in both intercalated and exfoliated nanocomposites. Moreover, exfoliated nanocomposites yield larger elastic moduli compared to intercalated ones despite the less significant modulus variation in terms of the interphase modulus as mentioned earlier. More significantly, by increasing the staggering factor, exfoliated nanocomposites demonstrate dramatic enhancements of relative modulus $E_{c} / E_{m}$ (maximum up to 2.25 for $E_{i}=2 E_{m}$ ), Fig. 9(a). When $f_{s}=1$, the relative modulus $E_{c} / E_{m}$ has achieved an improvement of over $25 \%$ in comparison with those at $f_{s}=0$. On the other hand, the relative moduli of intercalated nanocomposites are not greatly altered in magnitude, signifying the independent effect of the staggering factor, Fig. 9(b). In fact, the deformation of the PP matrix can be mainly constrained by the surrounding clay particles as the rigid reinforcements. For the exfoliated RVE models at both fixed clay aspect ratio and clay content, staggering effect can make a substantial contribution to more restrictions on the PP matrix as opposed to the largely unaffected matrix areas in the stack pattern of intercalated structures, when subjected to the same macroscopic strain. Accordingly, the efficiency of modulus enhancement is greatly promoted for exfoliated structures by increasing the staggering factor. Nevertheless, in the case of the intercalated RVE models, their lateral distance $(c=190 \mathrm{~nm})$ is 10 times larger than that for the exfoliated ones $(c=19 \mathrm{~nm})$. The constraints resulting from clay particles as well as the staggering 
effect are tremendously diminished with a much larger deformation occurring in the PP matrix. Hence, the staggering factor evidently becomes less significant for intercalated nanocomposites. This finding is in good agreement with the previous numerical results [15], which indicates that the elastic moduli of nanocomposites could be affected not only by the staggering factor but also the lateral distance between aligned clay platelets.

\subsection{Effect of RVE patterns on elastic modulus}

In order to study the clay dispersion effect on the overall mechanical behaviour of nanocomposites, the influence of three aforementioned RVE patterns on the elastic modulus with $W_{f}=5 \mathrm{wt} \%$ and $t_{i}=2 t$ are presented in Fig. 10 . The random pattern is very close to the real morphology of nanocomposites apart from the assumption of well-aligned structures and constant clay aspect ratios. It is noticeable that the relative modulus $E_{c} / E_{m}$ for exfoliated nanocomposites is in the following order

$$
\left(\frac{E_{c}}{E_{m}}\right)_{S T K}<\left(\frac{E_{c}}{E_{m}}\right)_{R A D}<\left(\frac{E_{c}}{E_{m}}\right)_{S T G(f s=1)}
$$

A different relationship for intercalated nanocomposites can be expressed as

$$
\left(\frac{E_{c}}{E_{m}}\right)_{S T K} \approx\left(\frac{E_{c}}{E_{m}}\right)_{R A D} \approx\left(\frac{E_{c}}{E_{m}}\right)_{S T G(f s=1)}
$$

\subsection{Comparisons between RVE modelling, experimental and theoretical results}

RVE modelling work using the stack pattern $\left(t_{i}=2 t\right)$ is compared with the experimental data obtained from PP-Hom H380F based nanocomposites (WR=1:2) [25] and the composites theories in terms of the intercalated and exfoliated structures,

Figs. 11 and 12. Numerical simulation of exfoliated nanocomposites $(L / t=100)$ display a narrow band of results amongst all RVE models with three various interphase moduli though the higher interphase modulus can result in a slightly greater modulus enhancement of nanocomposites, Fig. 11. Halpin-Tsai model and 
modified rule of mixture $(\mathrm{MRF}=0.66$ [26]) might show the upper bound of exfoliated nanocomposites, whereas the modified Halpin-Tsai model $(\mathrm{MRF}=0.66$ [26]) and HuiShia model generate relatively close predictions with the numerical results. All the theoretical models overestimate the relative modulus $E_{c} / E_{m}$ in comparison with the numerical counterparts. It is implied that the conventional micromechanical models for particulate composites might be no longer applicable to predict the stiffness of such well exfoliated nanocomposites. Due to the manufacturing difficulty in achieving the full exfoliation in PP/organoclay nanocomposites, the experimental data for exfoliated structures of such nanocomposites are not available up to date. However, the numerical approach still demonstrates a very similar enhanced relative modulus trend with increasing the clay content, which can be taken as a typical reference for any further study. Alternatively, numerical results of intercalated nanocomposites $(L / t=10)$ are greatly diverged with the interphase modulus $E_{i}=2 E_{m}$, giving the highest modulus enhancement, Fig. 12. The least modulus enhancement by less than $5 \%$ is clearly achieved for the intercalated RVE models with $E_{i}=0.5 E_{m}$. RVE models with $E_{i}=E_{m}$ have the best agreement with Hui-Shia model. Nevertheless, the experimental data appear to be more consistent with those predicted by the modified Halpin-Tsai model $(\mathrm{MRF}=0.66)$, especially at higher clay content beyond $8 \mathrm{wt} \%$ while RVE models with $E i=2 E_{m}$ fit Halpin-Tsai model quite well. RVE models with $E_{i}=E_{m}$ underestimate the experimental data possibly due to the consideration of the complete stacks of layered intercalated structure for RVE numerical modelling. In fact, transmission electron microscopic (TEM) results show that the real morphology of PP-Hom H380F based nanocomposites ( $\mathrm{WR}=1: 2)$ is a mix of intercalated and exfoliated structures [25] despite the prevalence of intercalation. It is well understood that a certain level of exfoliation with relatively large aspect ratios of clay platelets 
can further promote the elastic modulus, which is neglected in the complete intercalated RVE models. In addition, based on the experimental data, the interphase modulus might be in the range of $E_{m}$ and $2 E_{m}$ but much closer to $E_{m}$, which quite differs from the general assumption of $E_{i}=2 E_{m}$ employed in the previous literature for modelling nylon 6/ clay nanocomposites [20]. This result can be attributed to the addition of low molecular weight MAPP as the compatibiliser to reduce the interphase modulus since their short molecular chains are highly mobilised adjacent to clay platelet surfaces and interlayer areas [15]. More importantly, the higher MAPP content due to the negative matrix plasticisation effect $[25,27,28]$ could further decrease the interphase modulus, thus damaging the modulus enhancement mechanism. Consequently, apart from the difficulty in the experimental measurement of interphase modulus, the use of parametric study still offers an essential insight for the qualitative evaluation of the interphase properties.

\section{Conclusions}

2-D three-phase RVE models with $3 \times 3$ array domains have been successfully established with well-aligned completely exfoliated and intercalated structures $(L / t=100$ and 10 , respectively) to predict elastic moduli of PP/organoclay nanocomposites. It is confirmed that exfoliated nanocomposites have much higher elastic moduli compared to those of intercalated counterparts. The influence of interphase properties on the modulus enhancement of exfoliated nanocomposites appears to be less significant than that for intercalated ones. The efficiency of modulus enhancement in intercalated nanocomposites does not heavily rely on the staggering factor and RVE patterns because of a much larger lateral distance between aligned clay platelets to promote the deformation of PP matrix. Comparisons of RVE modelling results with composite theoretical models and experimental data suggest 
that Hui-Shia and modified Halpin-Tsai models can still provide reasonable agreements with numerical and experimental results for intercalated nanocomposites. However, all the theoretical models overpredict the numerical results obtained from exfoliated nanocomposites. Due to the presence of MAPP as a compatibiliser, the empirical relationship of $E_{m}<E_{i}<2 E_{m}$ has been found for the interphase modulus of intercalated PP/organoclay nanocomposites.

\section{Acknowledgements}

The support for this research provided by Tertiary Education Commission (TEC), New Zealand and the Foundation for Research, Science and Technology (FRST), New Zealand under the FRST grant \#UOAX 0406 is gratefully acknowledged.

\section{References}

[1] A. Okada, M. Kawasumi, A. Usuki, Y. Kojima, T. Kurauchi, O. Kamigaito, Synthesis and properties of nylon-6/clay hybrids, In: D.W. Schaefer, J.E. Mark (Eds.), Polymer based molecular composites: MRS Symposium Proceedings, Pittsburgh, 1990, vol. 171, pp. 45-50.

[2] Y. Kojima, A. Usuki, M. Kawasumi, A. Okada, Y. Fukushima, T. Kurauchi, O. Kamigaito, J. Mater. Res. 8 (1993) 1185-1189.

[3] J.W. Gilman, Appl. Clay Sci. 15 (1999) 31-49.

[4] R.A. Vaia, G. Price, P.N. Ruth, H.T. Nguyen, J. Lichtenhan, Appl. Clay Sci. 15 (1999) 67-92.

[5] P.B. Messersmith, E.P. Giannelis, J. Polym. Sci. A 33 (1995) 1047-1057.

[6] W. Lertwimolnun, B. Vergnes, Polymer 46 (2005) 3462-3471.

[7] N. Hasegawa, M. Kawasumi, M. Kato, A. Usuki, A. Okada, J. Appl. Polym. Sci. 67 (1998) 87-92.

[8] Y. Wang, F.B. Cheng, K.C. Wu, J. Appl. Polym. Sci. 93 (2004) 100-112. 
[9] J.C. Halpin, J.L.Kardos, Polym. Eng. Sci. 16 (1976) 344-352.

[10] G.P. Tandon, G.J.Weng, Polym. Compos. 5 (1984) 327-333.

[11] T.D. Fornes, D.R. Paul, Polymer 44 (2003) 4993-5013.

[12] N. Sheng, M.C. Boyce, D.M. Parks, G.C. Rutledge, J.I. Abes, R.E. Cohen, Polymer 45 (2004) 487-506.

[13] C.Y. Hui, D. Shia, Polym. Eng. Sci. 38 (1998) 774-782.

[14] D. Shia, C.Y. Hui, S.D. Burnside, E.P. Giannelis, Polym. Compos. 19 (1998) $608-617$.

[15] L.J. Zhu, K.A. Narh, J. Polym. Sci. 42 (2004) 2391-2406.

[16] V.A. Buryachenko, A. Roy, K. Lafdi, K.L. Anderson, S. Chellapilla, Compos. Sci. Technol. 65 (2005) 2435-2465.

[17] K.S. Katti, D. Sikdar, D.R. Katti, P. Ghosh, D. Verma, Polymer 47 (2006) 403 414.

[18] F. Perrin-Sarazin, M.T. Ton-That, M.N. Bureau, J. Denault, Polymer 46 (2005) 11624-11634.

[19] J.J. Luo, I.M. Daniel, Compos. Sci. Technol. 63 (2003) 1607-1616.

[20] R.S. Fertig III, M.R. Garnich, Compos. Sci. Technol. 64 (2004) 2577-2588.

[21] W.J. Drugan, J.R.Wills, J. Mech. Phys. Solids 44 (1996) 497-524.

[22] I.M. Gitman, H. Askes, L.J. Sluys, Eng. Fract. Mech. 74 (2007) 2518-2534.

[23] D.A. Brune, J. Bicerano, Polymer 43 (2002) 369-387.

[24] D.H. Kim, P.D. Fasulo, W.R. Rodgers, D.R. Paul, Polymer 48 (2007) 53085323.

[25] Y. Dong, Multi-scale effects on deformation mechanisms of polymer nanocomposites: experimental characterisation and numerical study, $\mathrm{PhD}$ thesis, The University of Auckland, Auckland, New Zealand, 2008. 
[26] Y.P. Wu, Q.X. Jia, D.S. Yu, L.Q. Zhang, Polym. Testing 23 (2004) 903-909.

[27] Y. Dong, D. Bhattacharyya, Compos. Part A: Appl. Sci. Manuf. 39 (2008) 11771191.

[28] Y. Dong, D. Bhattacharyya, Mater. Sci. Eng. A: Struct. Mater. Prop. Microstruct. Process. 527 (2010) 1617-1622.

\section{List of Figures:}

Fig. 1. Schematic diagram of two typical morphologies of intercalated and exfoliated nanocomposites.

Fig. 2. Three types of RVE patterns: (a) stack, (b) staggering and (c) randomly distributed.

Fig. 3. (a) Discrete stack structure and (b) equivalent isotropic homogenised particle for intercalated RVE models [12]. $\left(d_{001}, d_{s}\right.$ and $d_{i}$ are interlayer spacing, silicate layer thickness and galley thickness, respectively).

Fig. 4. Comparisons of elastic moduli of intercalated clay platelets based on ROM Eq. (3) and interlayer spacing values of PP-Hom H380F based nanocomposites (weight ratio: $\mathrm{WR}=1: 2)$.

Fig. 5. Typical finite element mesh of $3 \times 3$ array RVE models in the stack pattern $\left(t_{i}=2 t\right)$ : (a) exfoliated RVE model and (b) intercalated RVE model.

Fig. 6. Effect of interphase properties on the relative moduli of nanocomposites in the stack pattern $\left(W_{f}=5 \mathrm{wt} \%\right)$ : (a) exfoliated nanocomposites and (b) intercalated nanocomposites.

Fig. 7. Axial stress (a) and axial strain (b) distributions of exfoliated RVE models along the mid-plane $\mathrm{XZ}$ in the stack pattern $\left(W_{f}=5 \mathrm{wt} \%, t_{i}=2 t\right)$.

Fig. 8. Axial stress (a) and axial strain (b) distributions of intercalated RVE models along the mid-plane $\mathrm{XZ}$ in the stack pattern $\left(W_{f}=5 \mathrm{wt} \%, t_{i}=2 t\right)$. 
Fig. 9. Effect of staggering factor on the relative moduli: (a) exfoliated nanocomposites and (b) intercalated nanocomposites $\left(W_{f}=5 \mathrm{wt} \%, t_{i}=2 t\right)$.

Fig. 10. Effect of RVE patterns on the relative moduli of exfoliated and intercalated nanocomposites $\left(W_{f}=5 \mathrm{wt} \%, t_{i}=2 t\right)$. Symbols of "STK", 'STG" and "RAD" are denoted as the stack, staggering $\left(f_{s}=1\right)$ and randomly distributed patterns, respectively. “ex" and "in" represent the exfoliated and intercalated RVE models.

Fig. 11. Comparisons of relative moduli of exfoliated nanocomposites between RVE modelling results $\left(L / t=100\right.$ and $\left.t_{i}=2 t\right)$ and composite theoretical models.

Fig.12. Comparisons of relative moduli of intercalated nanocomposites between RVE modelling results $\left(L / t=10\right.$ and $\left.t_{i}=2 t\right)$, experimental data and composite theoretical models. 


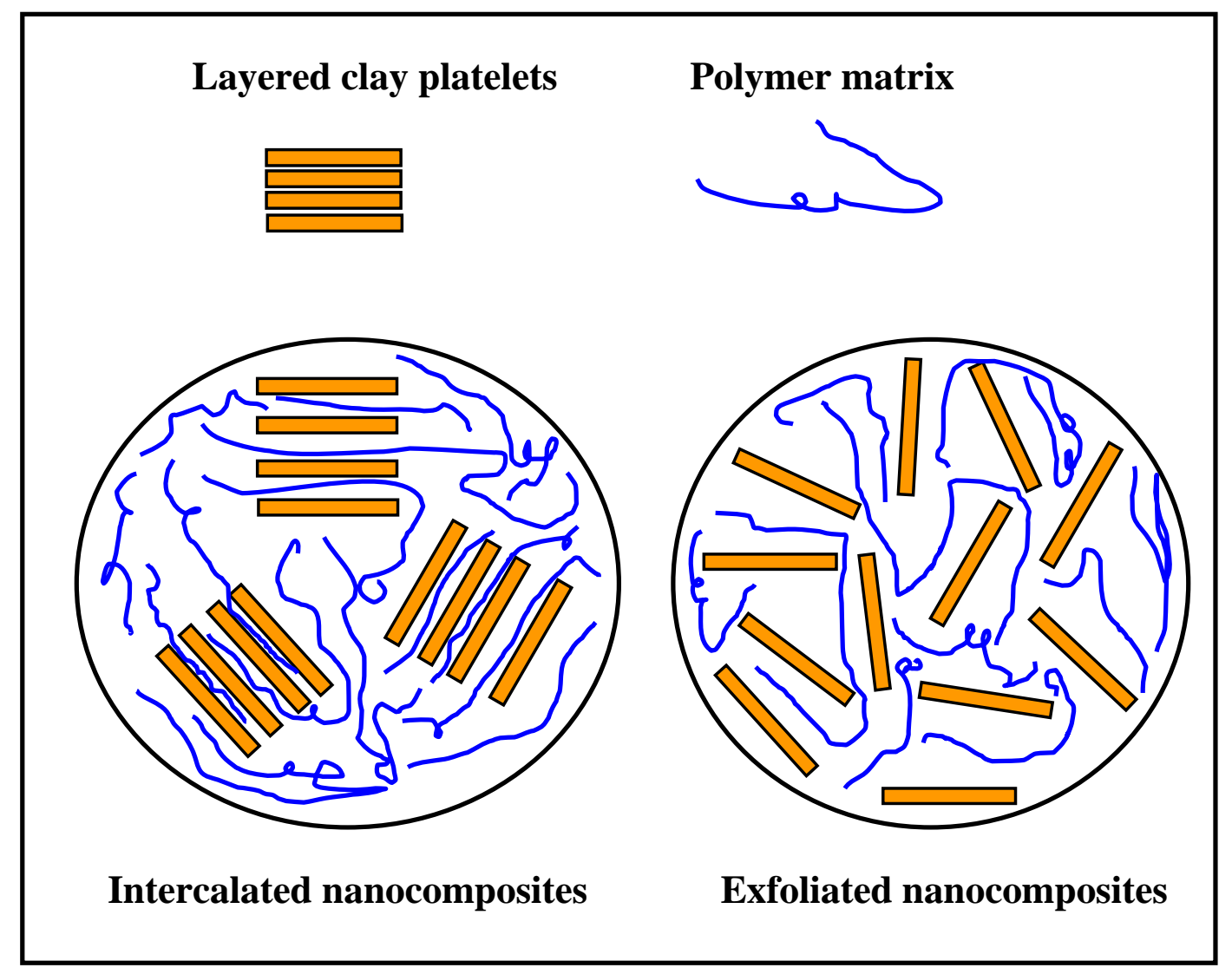

Fig. 1. 


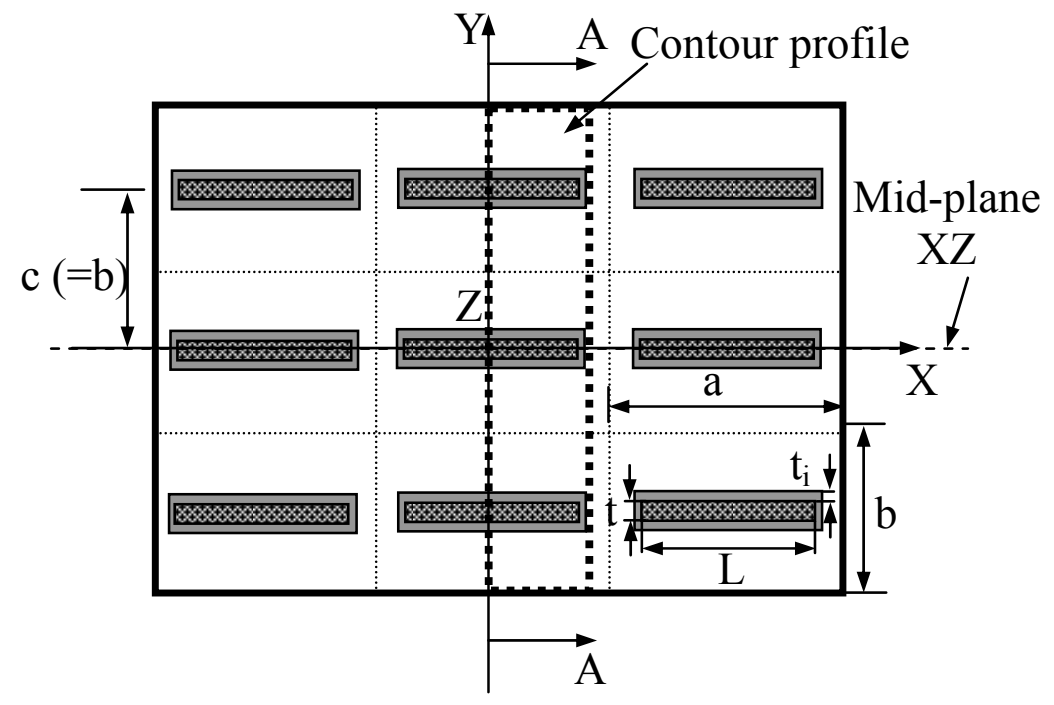

(a)

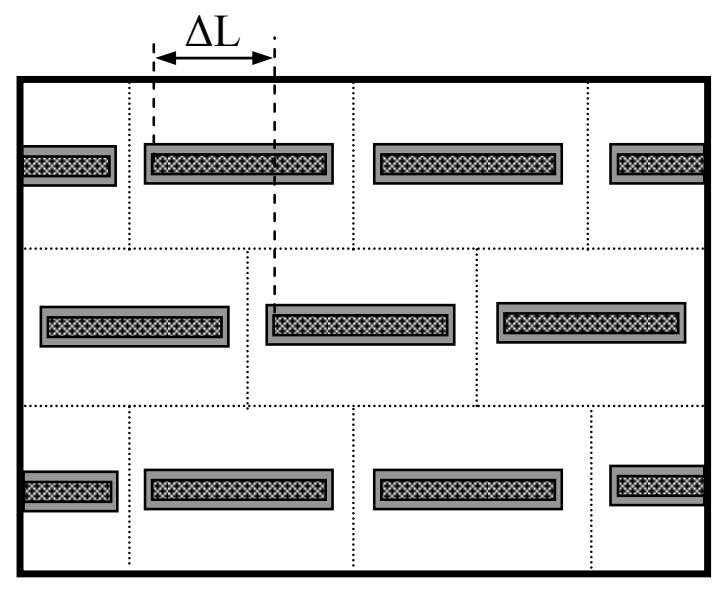

(b)

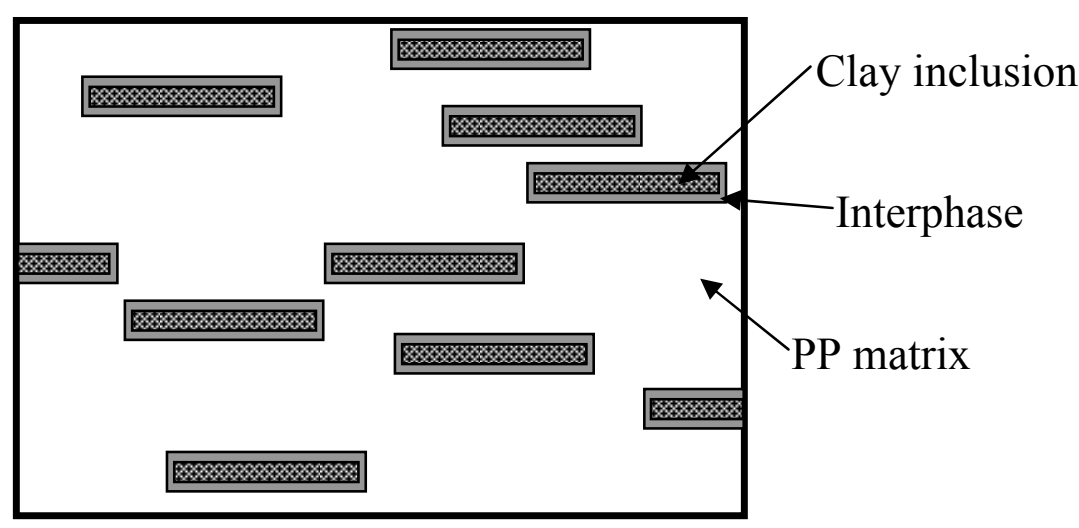

(c)

Fig. 2.

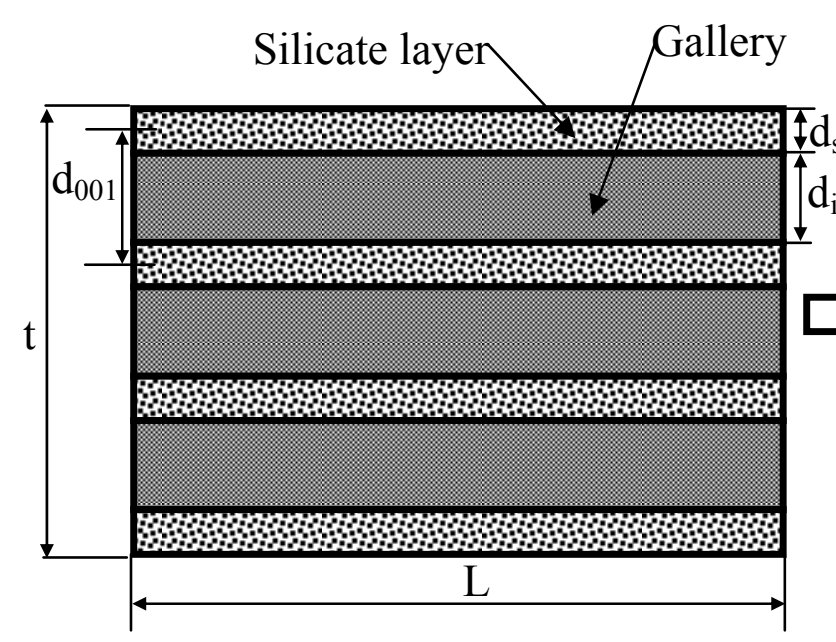

(a)

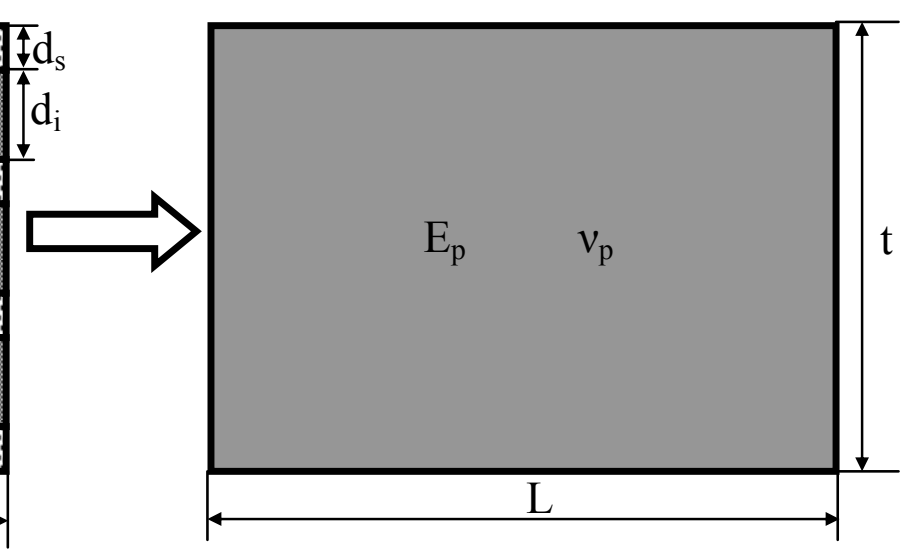

(b)

Fig. 3. 


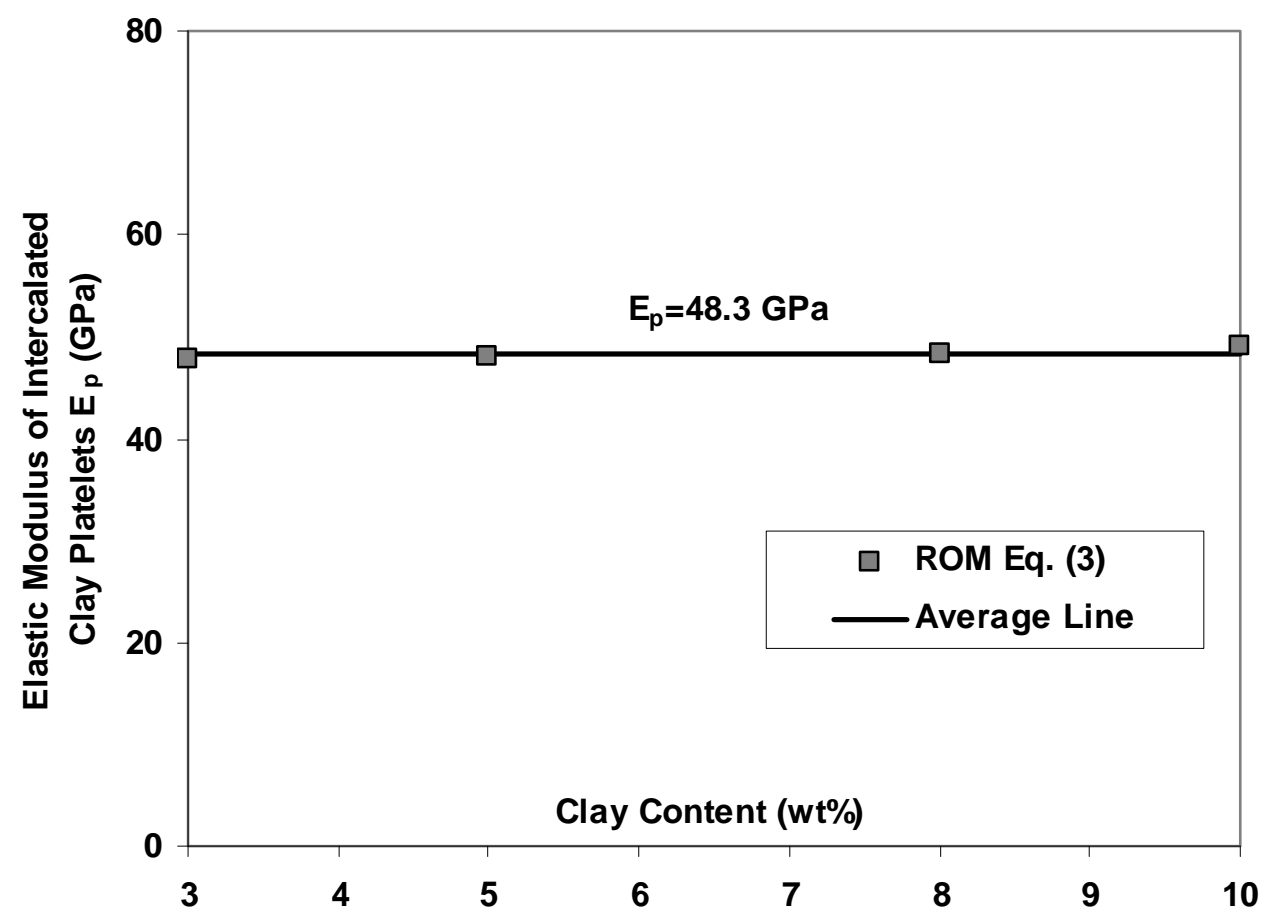

Fig. 4.

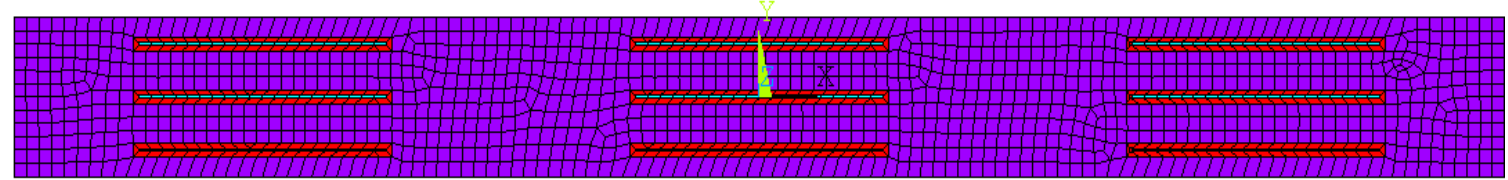

(a)

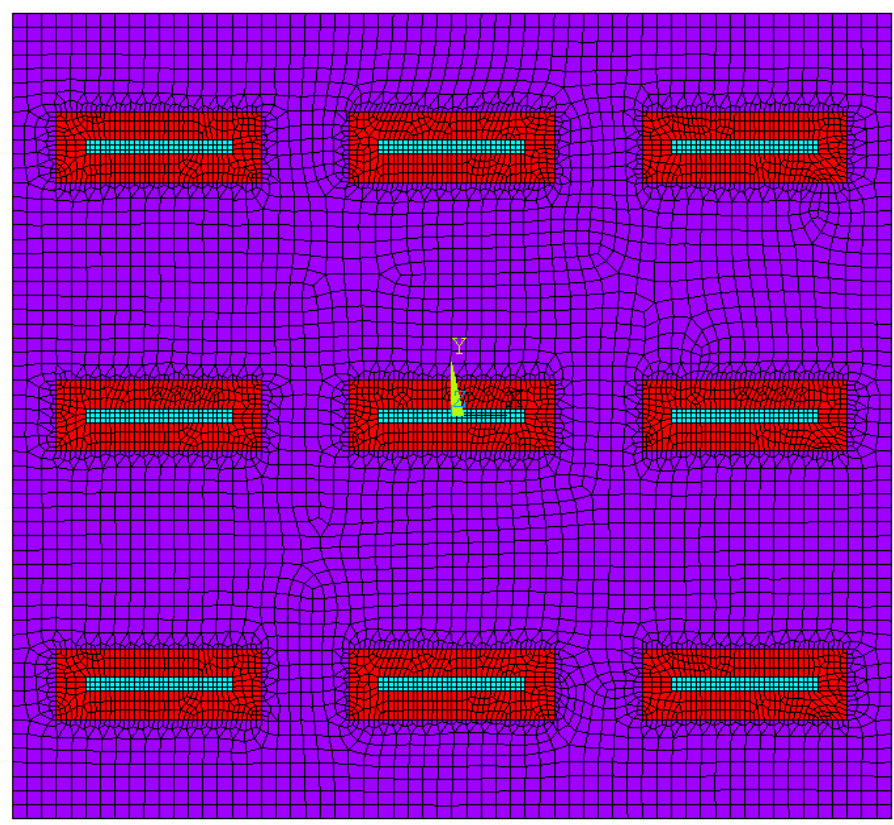

(b)

Fig. 5. 

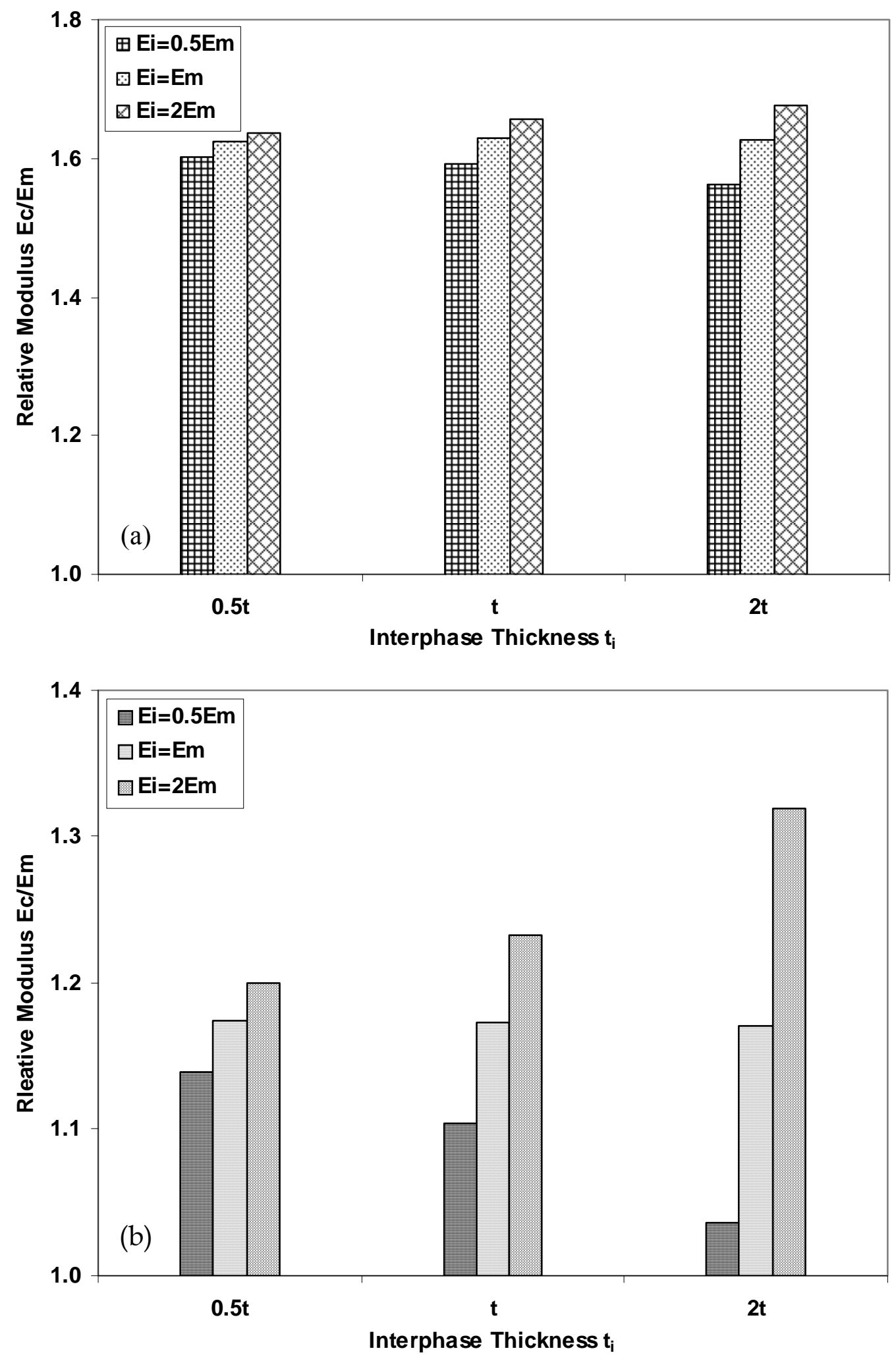

Fig. 6. 


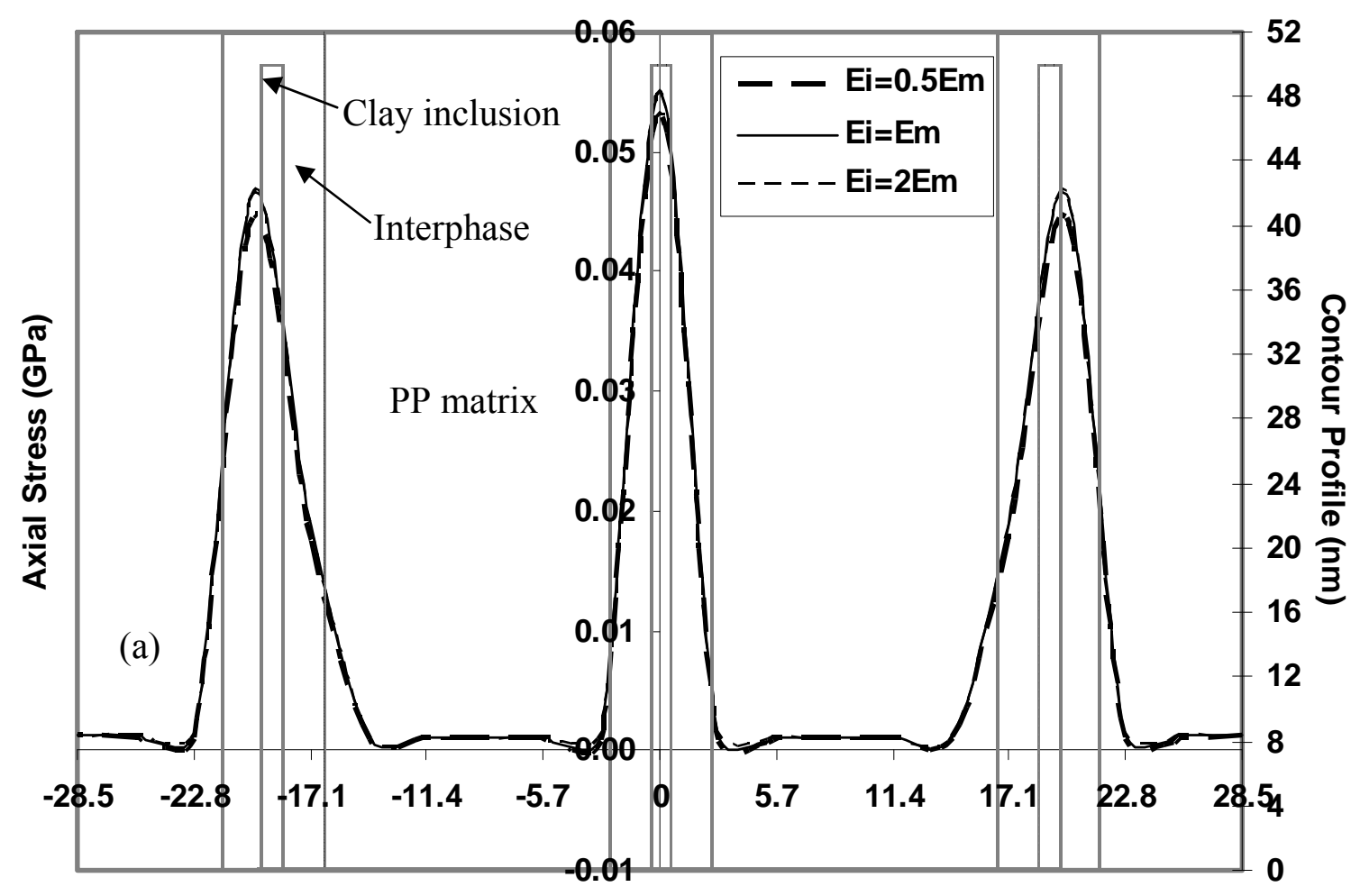

Distance from Mid-Plane XZ (nm)

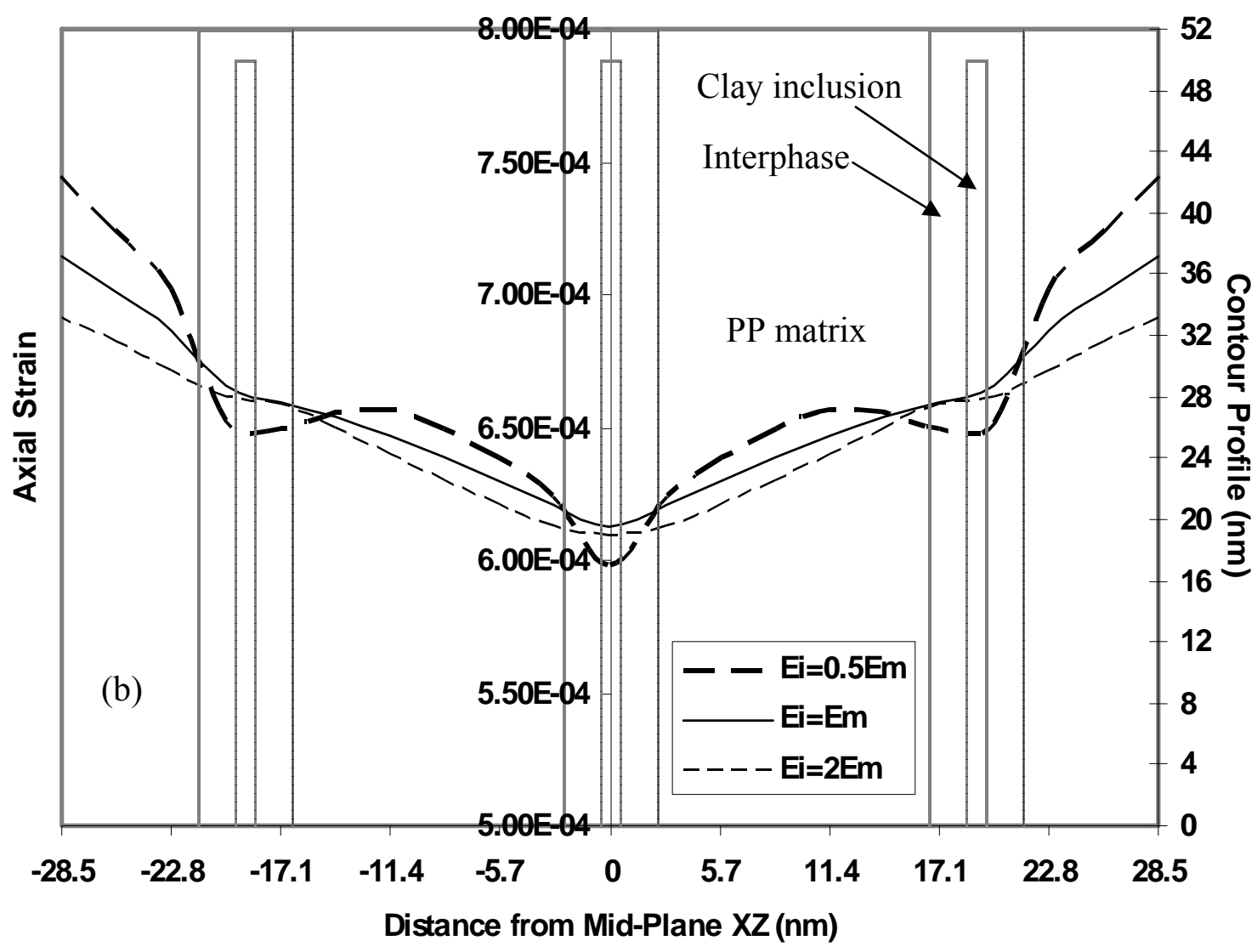

Fig. 7. 

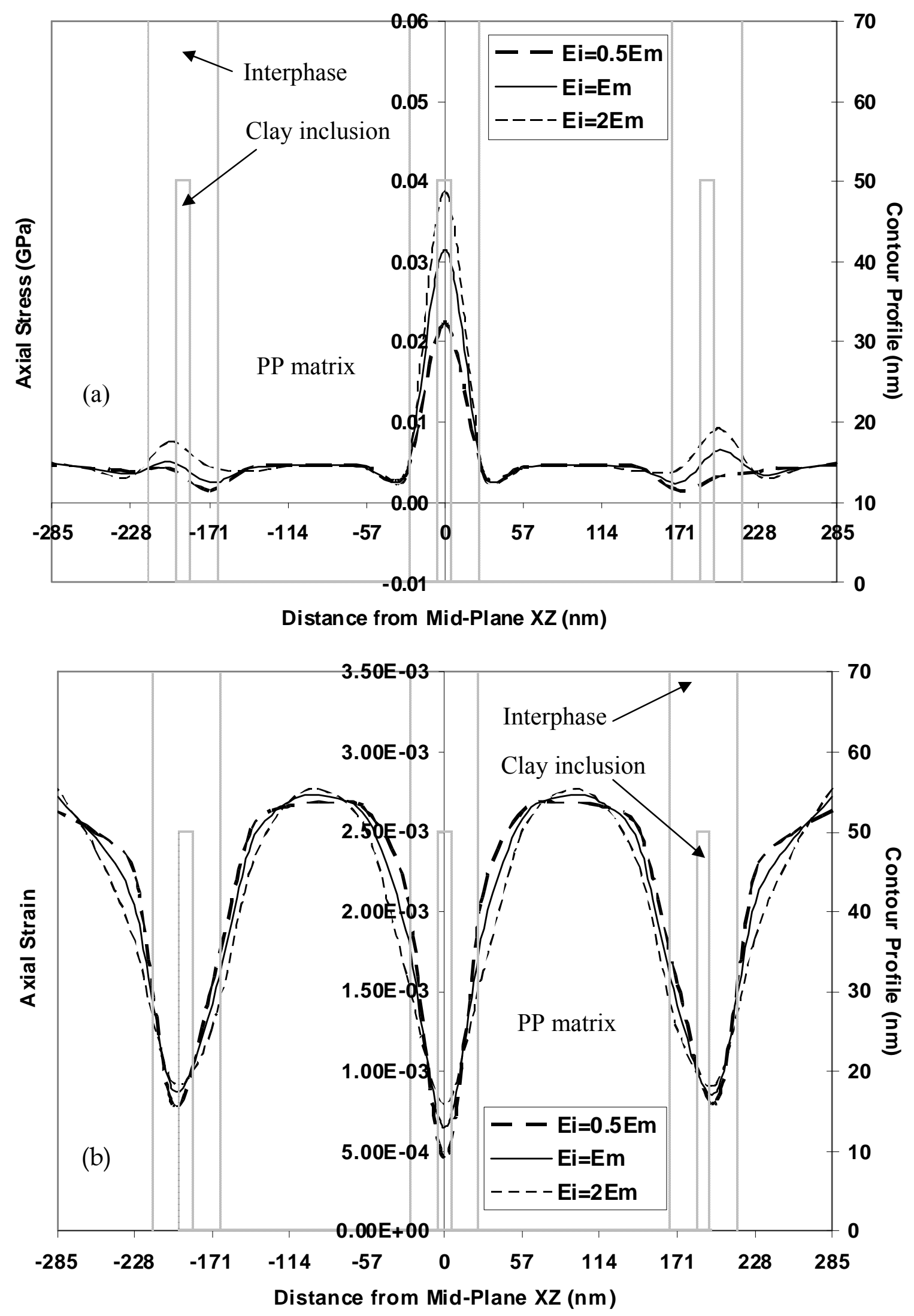

Fig. 8. 

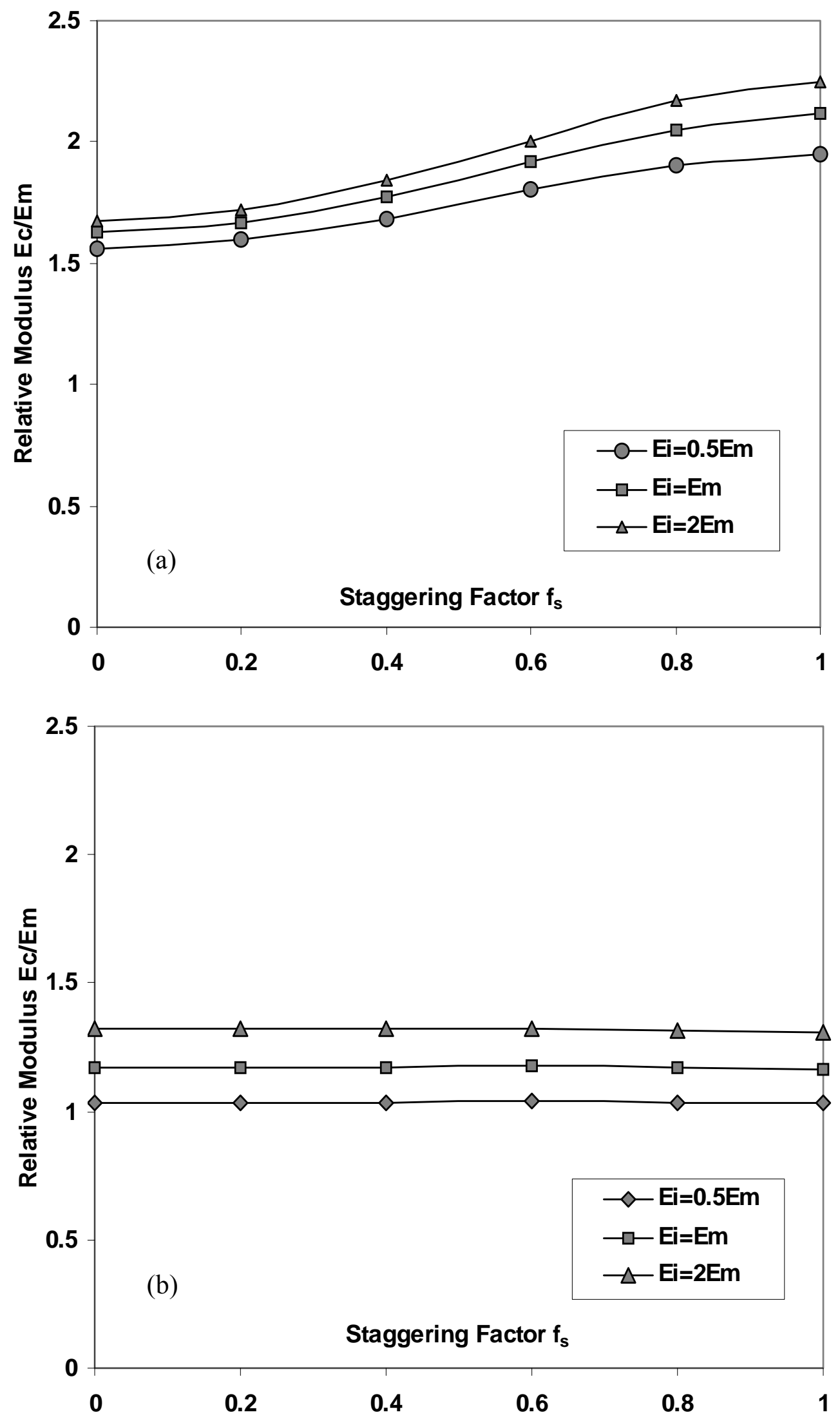

Fig. 9. 


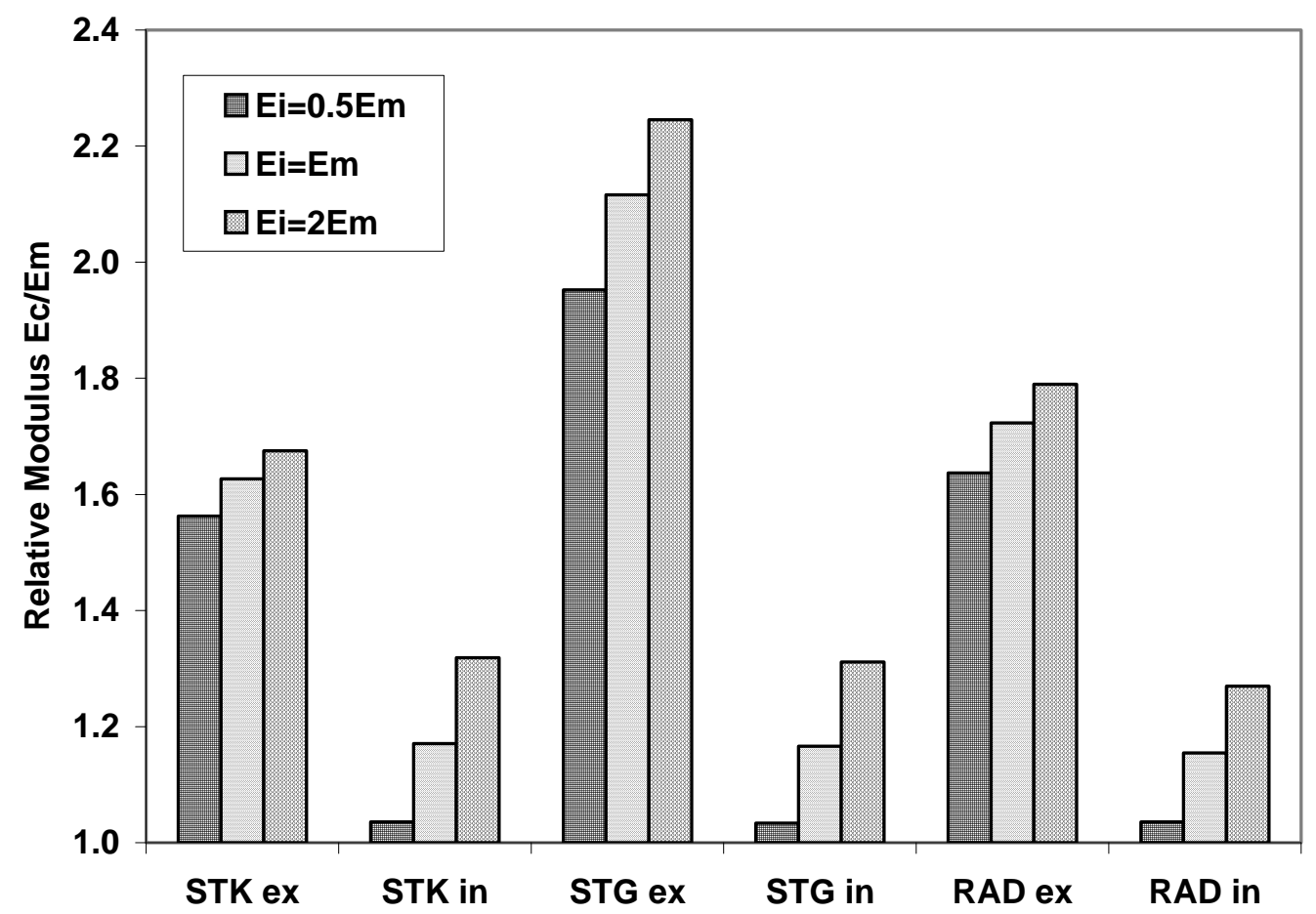

Fig.10.

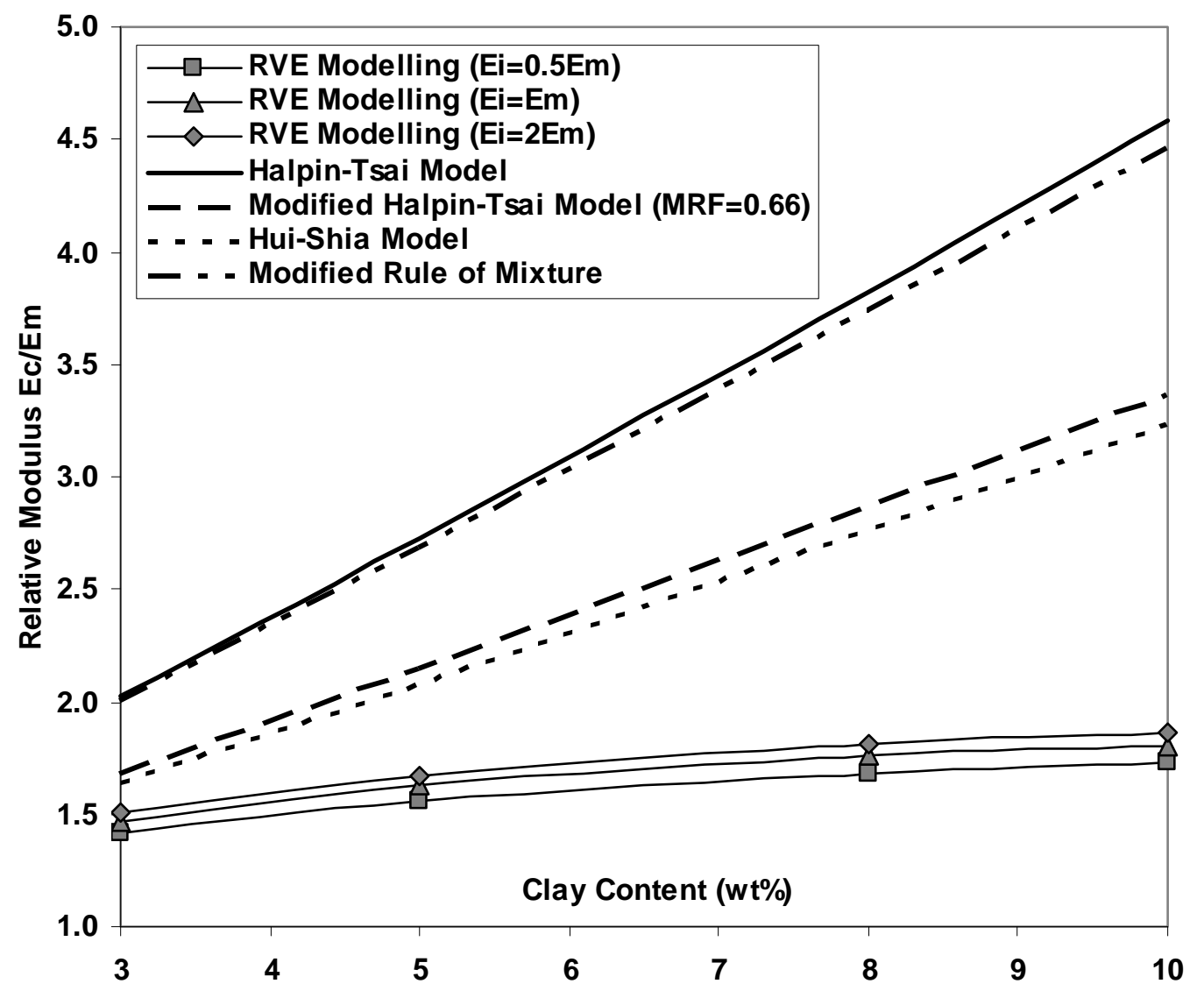

Fig. 11. 


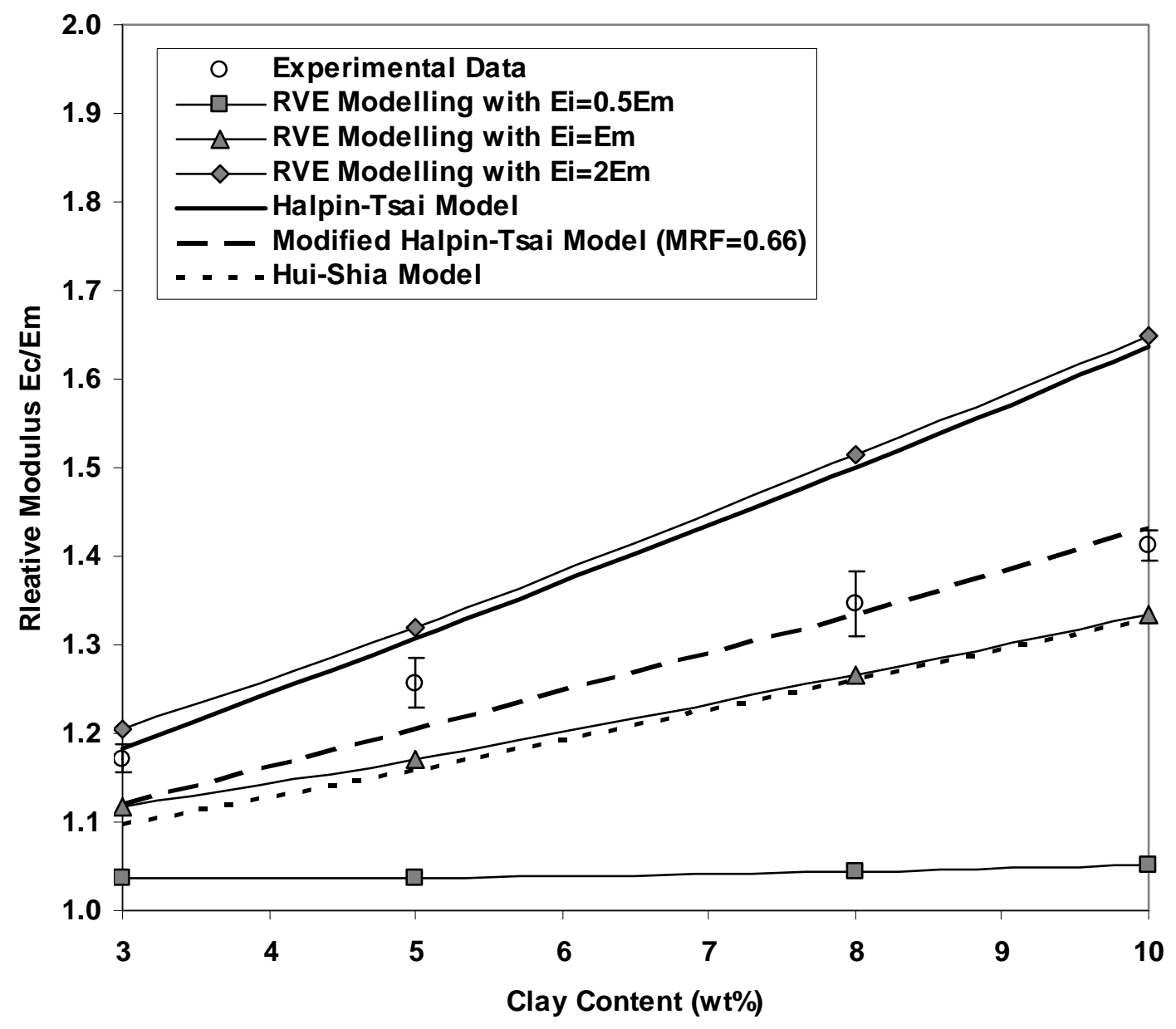

Fig. 12. 
Table 1 Interlayer spacing values $\mathrm{d}_{001}$ of PP-Hom H380F based nanocomposites $(\mathrm{WR}=1: 2)$ [25]

\begin{tabular}{cc}
\hline Clay content $(\mathrm{wt} \%)$ & $\mathrm{d}^{*}{ }_{001}(\mathrm{~nm})$ \\
\hline 3 & 3.73 \\
5 & 3.70 \\
8 & 3.68 \\
10 & 3.62 \\
\hline
\end{tabular}

$* d_{001}$ values were determined from the first peaks of XRD scans with the scan rate of $0.4 \%$ min at $2 \theta=2$ $10^{\circ} . d_{001}=3.56 \mathrm{~nm}$ for raw organoclay powders.

Table 2 Constituent properties in RVE modelling of PP/organoclay nanocomposites

\begin{tabular}{|c|c|c|c|c|c|c|}
\hline Material parameter & PP matrix & Exfoliated clay & Intercalated clay & \multicolumn{3}{|c|}{ Interphase } \\
\hline Elastic modulus (GPa) & $1.76^{\mathrm{a}}$ & 178 & 48.3 & 0.88 & 1.76 & $3.52^{\mathrm{b}}$ \\
\hline Poisson's ratio & $0.35^{\mathrm{c}}$ & 0.20 & 0.26 & & $0.35^{\mathrm{c}}$ & \\
\hline Density $^{\mathrm{d}}\left(\mathrm{g} / \mathrm{cm}^{3}\right)$ & 0.9 & 1.8 & 1.8 & & 0.9 & \\
\hline
\end{tabular}

Note: ${ }^{a}$ Experimental data of neat PP-Hom H380F, ${ }^{b}$ Ref. [20], ${ }^{c}$ Ref. [11] and ${ }^{d}$ material data sheet. 